\title{
Design and Implementation of Standard DICOM Interface Module
}

\author{
Am Suk $\mathrm{Oh}^{1}$, Oh Hyun Kwon ${ }^{2}$ and Gwan Hyung Kim ${ }^{2}$ \\ ${ }^{1}$ Department of Media Engineering, Tong-Myoung University, \\ Sinsun Ro 179, Nam-gu, Busan 608-711, Korea \\ ${ }^{2}$ Department of Computer Engineering, Tong-Myoung University, \\ Sinsun Ro 179, Nam-gu, Busan 608-711, Korea \\ asoh@tu.ac.kr,ohkwon@tu.ac.kr,ghkim@tu.ac.kr
}

\begin{abstract}
DICOM (Digital Imaging and Communications in Medicine) which is standard of PACS(Picture Archiving Communication System) is set up as draft international standard for the purpose of collection and exchange the data and image among main medical display equipments. Medical display equipments is normally utilized as connected feature with surgery monitoring devices like endoscope, X-lay camera. However, most of medical surgery monitoring devices are not adaptable for DICOM, thus it is diffcult to connect data thru PACS. We suggest the connection methodology for PACS and medical surgery monitoring devices by implementation of HL7 interface which can share the medical information with DICOM. The standard DICOM interface module suggested in this thesis creates DICOM IOD (Information Object Definition) information class according to the checkup information received by HIS (Hospital Information System) and also creates standard DICOM format after blending with surgery monitoring data.
\end{abstract}

Keywords: HIS, DICOM, HL7

\section{Introduction}

Currently most of latest medical image systems support the image by the international standard methodology. Many hospitals introduce PACS for getting the new type of image as standard DICOM grow up continuously. These days, PACS support the connection with DICOM, for example, these PACS can connect digital image devices like DR, CR using at surgery, internal medicine, and checkup section [1,8]. Especially in case of surgery, various type of medical surgery monitoring devices like endoscope, X-ray camera are utilized as the form of connection with existing PACS.

But, as yet, these medical surgery monitoring devices do not follow the standard format of HL7, DICOM which are standard medical information, so it can't support interconnection among medical surgery monitoring devices and patient data like archiving and managing image which are required in hospital information system. Thus, we designed and implemented interface module which can convert into DICOM form for the medical surgery monitoring devices. 


\section{Related Study}

The study concerning the application of the DICOM standard is as follows.

First of all, on the study "XML based module development for the conversion into DICOM ECG data from Non-DICOM ECG data" it suggests PACS connection thru XML-based DICOM converter as a digitalized method of living body signal information (ECG, EEG, BP etc.) $[2,8]$. But in case of living body signal, it is hard to make conclusion as image connected with medical image or PACS, and most of living body signal is defined as HL7 text based standard, thus can't suggest the DICOM application from non-DICOM devices.

Secondly, on the study "DICOM and non-DICOM interface methodology for the connection of PACS and the radiographic inspection devices", it implemented interface for the DICOM format conversion from specific non-DICOM CR devices [3, 8]. Although, this study has the way to connect with PACS after converting DICOM format from non-DICOM image data, it might be hard to have flexible PACS connection as it get and pass individual information process by using special software like Patient Terminal. Considering above study, we focused on the standardization of moving images of PACS.

\section{Design and Implementation}

\subsection{Configuration of system}

We designed and implemented core function module for the PACS application of medical surgery monitoring devices. We constructed standard communication using HL7 and DICOM among DICOM standard interface which operate as software module in medical surgery monitoring devices and HIS (Hospital Information System) interface in PACS [4, 8].

\subsection{Environments of system implementation}

The software module related with DICOM is developed under the dcm4che-2.0.25 sdk and dicom4j-0.0.9a sdk based system and software module related with HL7 is developed under HAPI $1.2 \mathrm{sdk}$ adapted system [5-8].

We used 7EDIT HL7 Tool for creation and test of HL7 message and also utilized RuboMedical DICOM Viewer for the validation of DICOM File. Implemented DICOM standard module was applied on DreamRay 60F which can process real time based monitoring thru mini X-Ray camera in case of implant surgical procedure and PAC server was tested under the environment of window XP operating system.

\subsection{System design and implementation}

(1) HL7 Interface

DICOM standard is comprised as a type of pair. They are "Information Object Definition (IOD)"which is visual information for storage and communication, and the other one is" 
DICOM Message Service Element (DIMSE)"which is command language. IDO includes text data and visual data related with decoding depending upon the regulation of visual information. Visual data is acquired from medical visual devices and text data is from HIS (Hospital Information System).[8] HIS system in PCS is required for the connection with DICOM data. Thus, we implemented HIS interface which can process HL7 message for the creation of object "DICOM IOD".

HIS Interface deal with HL7 message which perform creation of DICOM IOD object, connection with PACS. It targets HIS fulfilled HL7 standard. We constructed database for the new patient's registration, treatment, inspection record using HL7 message and HIS information. It is composed of simple application for worklist-server and testing based on HL7 2.5 message model. However it does not include service function (hospitalization, discharging hospital, treatment) of patients.

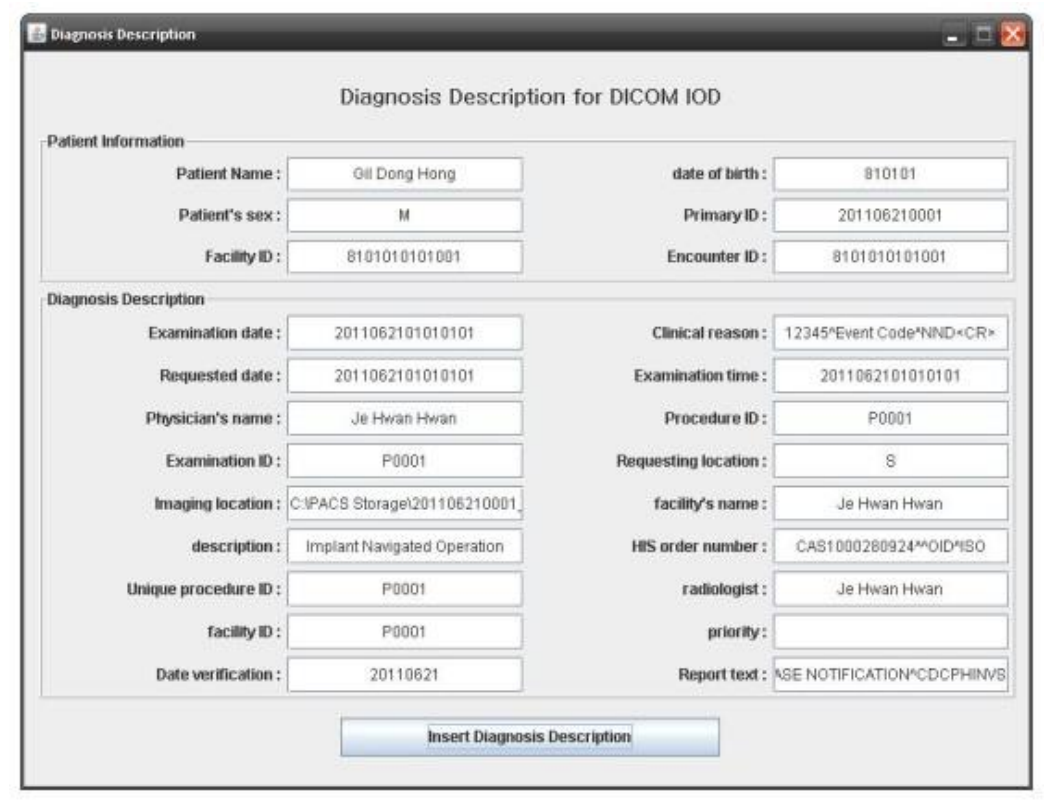

Figure 1. HIS Interface application

Figure 1 shows OBR, OBX data registration interface of HIS Interface application. It create $\mathrm{ORU}^{\wedge} \mathrm{R} 01$ (Unsolicited transmission of an observation message) message, and register the message at HIS database thru HL7 worklist server [8].

(2) DICOM standard interface module

DICOM standard interface module is application bridge module which transfers image data in non-DICOM medical surgery monitoring devices to standard DICOM image storage server of PACS. The structure of standard DICOM interface module is shown in Figure 2. 


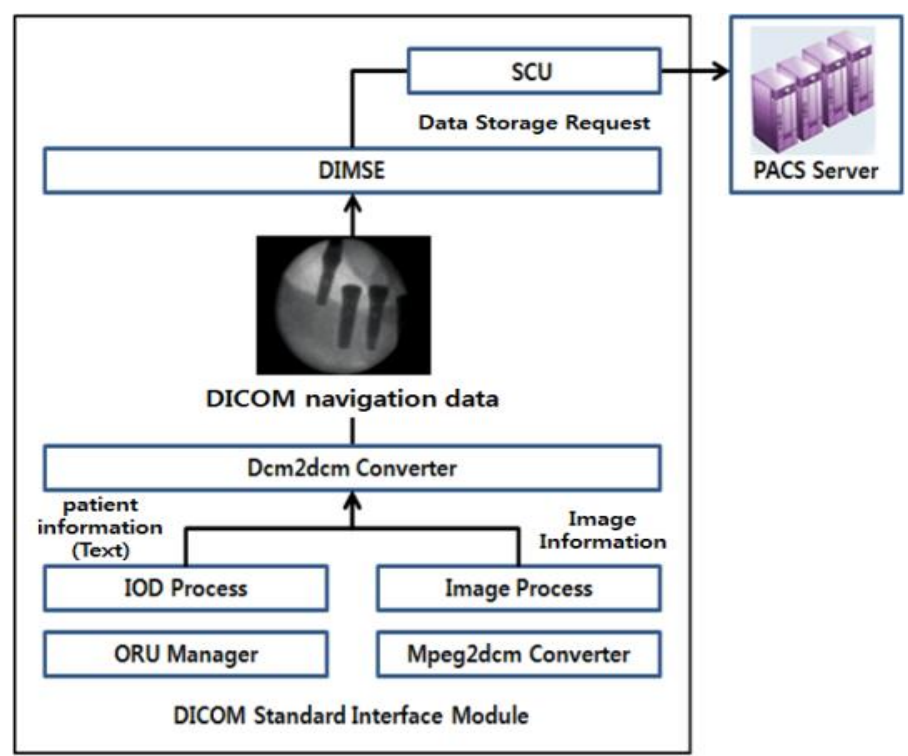

Figure 2. DICOM interface module

The operation of standard DICOM interface module implemented in this study is shown in Figure 3.

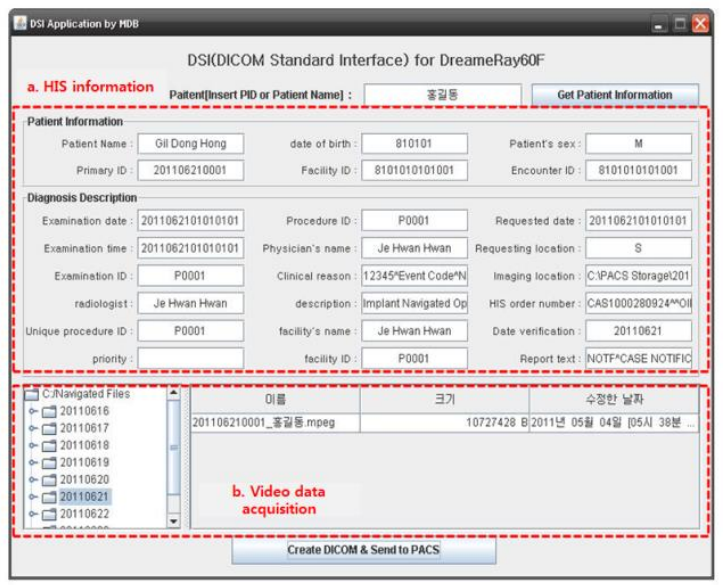

Figure 3. DICOM standard module application

First, standard DICOM interface module operated as software module in medical surgery monitoring devices request $\mathrm{ORU}^{\wedge} \mathrm{R} 30$ message transmission to the HIS server of PACS, and then it gets PID, OBR, OBX segment data of surgery patients as shown in Figure 3(a). Secondly, it gets the visual data which is taken picture during surgery time as shown in Figure 3(b). Third, using acquired data, it converts the Mpeg2 codec video into DICOM File via Mpeg2dcm Converter. It is not completed form as there is no DICOM Tag as yet.

Fourth, IOD Process received creates DICOM IOD object thru PID, OBR, OBX segment data according to DICOM/HL7. It also creates DICOM Tag which includes IOD object and visual data, and then it creates surgery monitoring visual data of standard DICOM. Fifth, created standard DICOM visual data is transmitted to PACS thru DICOM SCU (Service 
Class User) module and then it is stored by request. Figure 4 shows the picture of surgery monitoring visual data created from surgery monitoring devices.

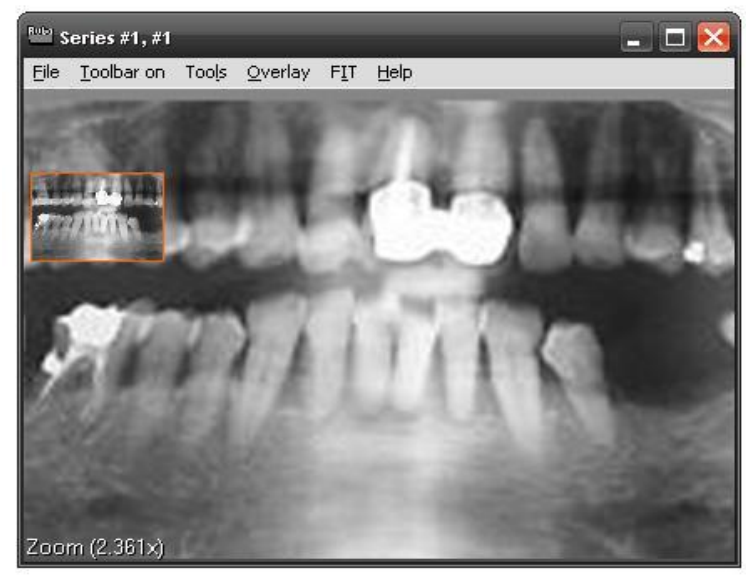

Figure 4. Picture of DICOM viewer

\section{Conclusion}

The standard DICOM interface module suggested in this thesis creates DICOM IOD information class according to the checkup information received by HIS and also creates standard DICOM format after blending with surgery monitoring data. Thus there's no need to construct extra management system and it makes possible to connect with HIS. i.e., store and manage the hospital patient's information. For the more, it can reduce system management cost as the method of PACS connection in hospital, and we expect the medical surgery including decode could be more efficient as surgery monitoring and medical video devices are operating on same place. The standard DICOM interface module should be applied to standard of PACS is set up as draft international standard for the purpose of collection and exchange the data and image among main medical display equipments. Especially in case of surgery, various type of medical surgery monitoring devices like endoscope, X-ray camera are utilized as the form of connection with existing PACS.

\section{Acknowledgements}

This work (Grants No. C0125015) was supported by Business for Cooperative R\&D between Industry, Academy, and Research Institute funded Korea Small and Medium Business Administration in 2013.

\section{References}

[1] lth Infoway Inc., "Electronic Health Record Infostructure (EHRi) Privacy and Security Conceptual Architecture", Version 1.1, (2005) June.

[2] B. Blazona and M. Koncar, "HL7 and DICOM based integration of radiology departments with healthcare enterprise information systems", International Journal of Medical Informatics, vol. 76, no. 3, (2007), pp. 425432.

[3] dicom4j-0.0.9a SDK, http://dicom4j.sourceforge.net.

[4] HAPI $1.2 \mathrm{sdk}$, http://hl7api.sourceforge.net.

[5] DreamRay 60F, http://www.dreamray.co.kr. 
[6] B. Blazona and M. Koncar, "HL7 and DICOM based integration of radiology departments with healthcare enterprise information systems", International Journal of Medical Informatics, vol. 76, Supplement 3, (2007) December, pp. S425-S432.

[7] M. Prinz, G. Fischer and E. Schuster, "The JAVA-based DICOM query interface DicoSE Original Research Article", International Journal of Medical Informatics, vol. 74, no. 2-4, (2005), pp. 325-333.

[8] A. S. Oh, O. H. Kwon and G. H. Kim, "A Study on DICOM Standardization of Monitoring Equipment for Medical Surgery", International Workshop on SERSC, Advanced Science and Technology Letters, vol. 33, (Bioscience and Medical Research 2013), Jeju, Korea (2013) December 11-13, pp. 49-52.

[9] C. Y. Park, J. H. Lim and S. Park, "ISO/IEEE 11073 PHD standardization of legacy healthcare devices for home healthcare services", IEEE International Conference on Consumer Electronics (ICCE), IEEE, (2011), pp. 547-548.

[10] J. -C. NAM, et al., "Design and Development of a u-Health System Based on the ISO/IEEE 11073 PHD Standards", 2011 17th Asia-Pacific Conference on Communications (APCC), IEEE, (2011), pp. 789-793.

\section{Authors}

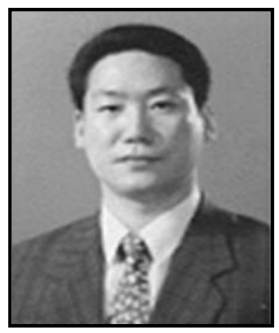

\section{Am-Suk Oh}

He received Ph.D. degree at the computer engineering of Pusan National University. He is currently with the Department of Media Engineering, Tongmyong University as Professor. His research interests are Healthcare System, Medical Information System, Big Data.

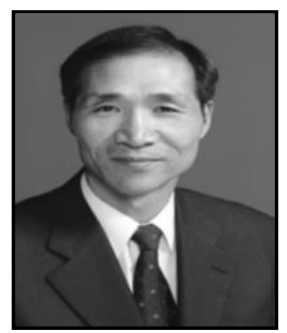

\section{Oh-Hyun Kwon}

$\mathrm{He}$ is currently a professor in the Department of Computer Engineering, Tongmyong University in Korea. He received $\mathrm{Ph}$. D degree in 1989 at the Computer Engineering Department of ChungAng University. His research interests are System Software Component Based Development, Software Architecture.

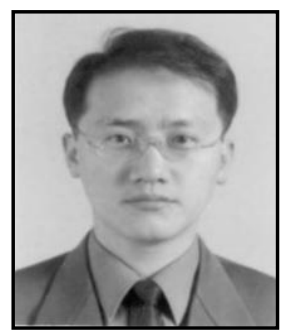

\section{Gwan-Hyung Kim}

$\mathrm{He}$ is received the Ph.D. degrees in electrical communication from Korea Maritime University in 2002. He is currently an assistant professor in Dept. of Computer Engineering, Tongmyong University. His research interests are intelligent control, signal processing, and robotics. 